\title{
Bioprospecção de macroalgas marinhas e plantas aquáticas para o controle da antracnose do feijoeiro
}

\author{
Guilherme Fernandes de Abreu, Viviane Talamini, Marciel João Stadnik
}

Departamento de Fitotecnia, Universidade Federal de Santa Catarina, CP 476, 88040-900 Florianópolis, SC, e-mail: stadnik@cca.ufsc.br. Autor para correspondência: Marciel J. Stadnik

Parte da Dissertação de mestrado do primeiro autor, apresentada à Universidade Federal de Santa Catarina.

Data de chegada:30/01/2006. Aceito para publicação em:26/01/2007

\section{RESUMO}

Abreu, G.F.; Talamini, V.; Stadnik, M.J. Bioprospecção de macroalgas marinhas e plantas aquáticas para o controle da antracnose do feijoeiro. Summa Phytopathologica, v.34, n.1, p.78-82, 2008

O objetivo deste trabalho foi testar o efeito local, residual e sistêmico, de extratos de 17 espécies de macroalgas marinhas e de duas plantas aquáticas, sobre a antracnose do feijoeiro. Para tanto, os espécimes foram coletados, identificados, secos em estufa $\left(50^{\circ} \mathrm{C} / 48\right.$ h), moídos e seus compostos extraídos com etanol. Plantas de feijoeiro (Phaseolus vulgaris cv. Uirapuru) foram cultivadas em vasos, em casa-de-vegetação. Os 19 extratos foram subdivididos e testados em duas etapas de seleção e comparação independentes, utilizando-se o delineamento inteiramente ao acaso, com cinco repetições (vasos com três plantas). As plantas foram pulverizadas com extratos na concentração de $50 \mathrm{mg}$ de peso $\mathrm{seco} / \mathrm{mL}$ quando apresentavam o primeiro trifólio expandido. Para verificar o efeito local, as plantas foram inoculadas com uma suspensão de $1,2 \times 10^{6}$ conídios $/ \mathrm{mL} 4$ horas após o tratamento, enquanto que para o estudo do efeito residual e sistêmico, as plantas foram inoculadas 7 dias após o tratamento. A severidade da antracnose foi avaliada 7 dias após a inoculação (dai) na planta inteira e no trifólio não tratado (efeito sistêmico), utilizandose uma escala de 1 a 9. As algas e plantas que reduziram significativamente a severidade da doença foram comparadas em experimento avaliado aos 7 e aos 12 dai. O extrato de Bryothamnion seaforthii apresentou efeito local, reduzindo em $35 \%$ a severidade da antracnose, enquanto o extrato de Ulva fasciata demonstrou efeito residual com redução de $22 \%$ na doença aos 12 dai. Somente os extratos de Lemna sp. e U. fasciata reduziram sistemicamente a severidade de doença aos 7 dai na ordem de 55 e 44\%, respectivamente, em relação à testemunha. $\mathrm{O}$ possível modo de ação desses extratos é discutido.

Palavras chave: Colletotrichum lindemuthianum, Phaseolus vulgaris, algas marinhas, plantas aquáticas, bioprospecção.

\section{ABSTRACT}

Abreu, G.F.; Talamini, V.; Stadnik, M.J. Bioprospecting of marine seaweeds and aquatic plants for controlling the bean anthracnose. Summa Phytopathologica, v.34, n.1, p.78-82, 2008

The goal of this work was to test the local, residual as well as systemic effect of extracts from 17 marine seaweeds and two aquatic plant species against the bean anthracnose. For that, specimens were collected, identified, dried into an oven at $50^{\circ} \mathrm{C}$ for $48 \mathrm{~h}$, ground to powder and their compounds extracted with ethanol. Bean plants (Phaseolus vulgaris cv. Uirapuru) were grown in pots under greenhouse conditions. The 19 extracts were shared into two independent groups for screening and comparison in a completely randomized design, with five replications (pot with 3 plants). Plants at the first trifoliolate leaf stage were sprayed with extracts at concentration of $50 \mathrm{mg}$ dry weight $/ \mathrm{mL}$. To assess the local effect, plants were inoculated with a suspension of $1.2 \times 10^{6}$ conidia/mL $4 \mathrm{~h}$ after the treatment, whereas to study the residual and systemic effects, inoculation was performed after 7 days. Disease severity was evaluated 7 days after inoculation (dai) on either whole plants or the non-treated leaf (systemic effect), using a scale from 1 to 9 . Seaweeds and plants which significantly reduced anthracnose were compared in a follow-up experiment evaluated at both 7 and 12 dai. The extract of Bryothamnion seaforthii revealed local effect reducing the anthracnose severity by $35 \%$. Ulva fasciata extract showed residual effect reducing the anthracnose at 12 dai by $22 \%$. Compared to control, only extracts of Lemna sp. and $U$. fasciata systemically reduced the disease severity at 7 dai by $55 \mathrm{e}$ $44 \%$, respectively. The possible mode of action of these extracts is discussed.

Additional keywords: Colletotrichum lindemuthianum, Phaseolus vulgaris, marine seaweeds, aquatic plants, bioprospecting.

As macroalgas marinhas são encontradas em uma grande diversidade de espécies no extenso litoral brasileiro. Apesar do grande potencial, este recurso natural ainda é pouco utilizado na agricultura. Por outro lado, em países da Europa e nos Estados Unidos, produtos à base de algas vêm sendo comercializados há várias décadas como bioestimulantes de plantas, fertilizantes e condicionantes de solo $(6,12)$.

A exploração destes organismos, e/ou dos seus metabólitos na proteção de plantas contra patógenos é recente. Preocupações com o meio ambiente e com o aumento dos custos de produção das lavouras devido à utilização de produtos químicos sintéticos têm intensificado o interesse pela busca de moléculas ativas que podem estar presentes nas algas marinhas (18).

Em diversos estudos a atividade antimicrobiana tem sido freqüentemente relatada, principalmente nas algas vermelhas e marrons $(1,4,9,20)$. Por exemplo, o extrato da alga Cystoseira tamariscifolia apresenta forte atividade contra os fungos Fusarium oxysporum e Verticillium albo-atrum, e contra a fitobactéria Agrobacterium tumefaciens (4). Alolaurinterol obtido a partir da espécie Laurencia obtusa inibe in vitro diversos fungos fitopatogênicos, entre eles, 
Ustilago violacea e F. oxysporum (8). Além da atividade direta contra microorganismos, alguns estudos confirmam a indução de resistência das plantas a doenças devido à aplicação de extratos de algas marinhas $(7,9)$.

Na prática, somente as algas marrons têm mostrado eficiência no controle de doenças de plantas, como por exemplo, a Laminaria digitata e Ascophyllum nodosum. Da L. digitata se extrai o polissacarídeo laminarina, que é capaz de elicitar respostas de defesa do hospedeiro (7). Atualmente, a laminarina é comercializada na Europa com o nome de Iodus $40^{\circledR}$ e utilizada para induzir resistência a doenças do trigo, tais como fusariose, oídio e septoriose (6). Por sua vez, o extrato da alga $A$. nodosum estimula a atividade de peroxidases e a síntese da fitoalexina capsidiol em plantas de pimentão, aumentando a resistência das plantas a Phytophtora capsici (9). Produtos de $A$. nodosum, geralmente puros ou misturados com fertilizantes vêm sendo comercializados em diferentes países como bioestimulantes e/ou bioprotetores de plantas contra doenças (18).

Com relação às algas marinhas verdes há, ainda, poucos estudos. Tem-se demonstrado que o extrato de Ulva fasciata é capaz de reduzir eficientemente o número de colônias do oídio em feijoeiro (15). Em outro estudo, a aplicação foliar de polissacarídeos de Ulva spp. protegeu alfafa contra a subseqüente infecção por Colletotrichum trifolii (5).
Diante dos resultados promissores e da carência de trabalhos que explorem o potencial das macroalgas marinhas no litoral do Brasil o presente estudo foi realizado. Para tanto, optou-se pelo patossitema Colletotrichum lindemuthianum - feijoeiro pois o controle da antracnose pela aplicação de fungicidas é uma prática dispendiosa. O controle alternativo por meio de aplicação de extratos de macroalgas marinhas pode vir a colaborar no manejo integrado e/ou ecológico desta doença de uma forma menos impactante ao agroecossistema. Duas plantas aquáticas também foram incluídas neste trabalho, devido a grande quantidade em que são encontradas na região do estudo.

Assim, com este trabalho objetivou-se verificar o efeito local, residual e sistêmico dos extratos de macroalgas marinhas e/ou plantas aquáticas no controle da antracnose do feijoeiro.

As algas foram coletadas entre outubro de 2002 e agosto de 2004, durante as horas de maré baixa, na zona intermarés, conforme recomendação de Magallanes et al. (13). As áreas de coleta foram os costões rochosos nos contornos das praias de Florianópolis (ilha de Santa Catarina), com coletas mais concentradas na praia de Ponta das Canas. Após cada coleta, as algas foram acondicionadas em sacos plásticos e transportadas imediatamente para o laboratório para lavagem e seleção. Foram coletadas 17 macroalgas marinhas e duas plantas aquáticas (Tabela 1).

Tabela 1. Efeito local, residual e sistêmico de extratos etanólicos de macroalgas marinhas e plantas aquáticas na severidade de antracnose em plantas de feijoeiro aos 7 dias após inoculação, nos ensaios 1 e 2 .

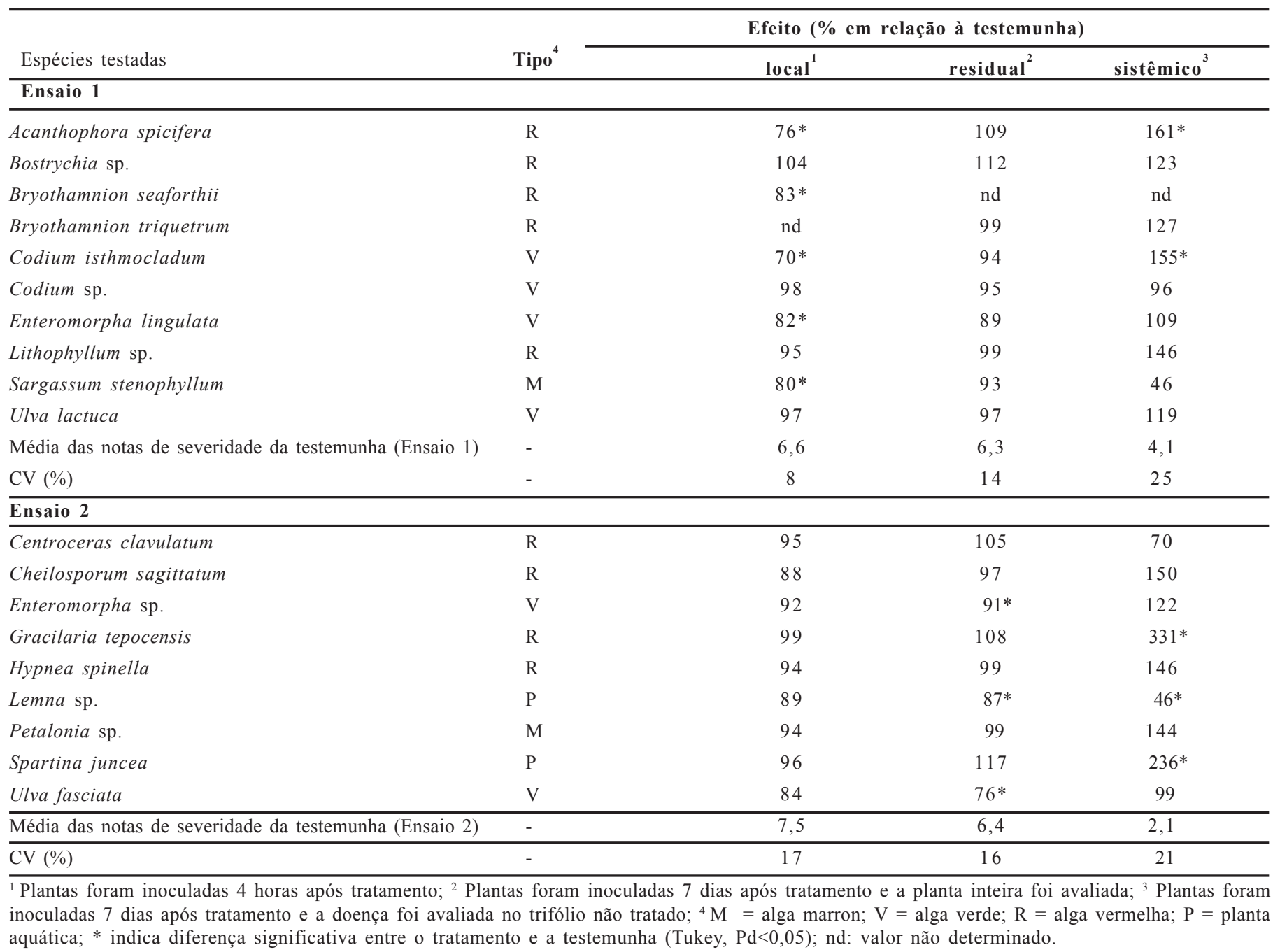


As algas e plantas aquáticas foram secas separadamente em estufa a $50^{\circ} \mathrm{C}$ por 48 horas. O material seco foi finamente triturado e o pó seco de cada espécime foi mantido em recipiente opaco na temperatura de $6^{\circ} \mathrm{C}$ até a extração.

Para extração dos compostos, adicionou-se etanol (98\%) ao pó seco, na relação 3,5:100 peso/volume. Após 24 horas, o extrato foi filtrado em papel filtro Whatman $\left(n^{\circ} 4\right)$, o etanol evaporado e a fração aquosa recolhida e quantificada. $\mathrm{O}$ extrato bruto foi diluído em água até a concentração de $50 \mathrm{mg}$ de peso seco/mL para ser utilizado no tratamento das plantas de feijoeiro.

Utilizou-se o isolado monospórico (Labfitop 001-03) da raça 73 de $C$. lindemuthianum (11). O inóculo consistiu de uma suspensão de conídios do fungo na concentração de $1,2 \times 10^{6}$ conídios $/ \mathrm{mL}$.

Sementes de feijoeiro (Phaseolus vulgaris L. cv. Uirapuru) foram semeadas em vasos plásticos $\mathrm{n}^{\circ} 3$ (capacidade 1,5 L), contendo uma mistura de solo e composto orgânico $(2: 1 ; \mathrm{v} / \mathrm{v})$ e irrigadas periodicamente, conforme as necessidades hídricas da cultura. Foram mantidas três plantas por vaso e cada vaso constituiu uma repetição.

As aplicações dos extratos e a inoculação foram realizados com auxílio de uma pistola acoplada a um motocompressor de ar (Marca Schulz, 25lbf $/ \mathrm{pol}^{2}$ ). Para testar o efeito local, os extratos foram pulverizados até ponto de escorrimento quando as plantas apresentavam o primeiro trifólio completamente desenvolvido e a inoculação destas plantas foi realizada imediatamente após a secagem do extrato (aproximadamente 4 horas). Para testar o efeito residual e sistêmico, as plantas foram também tratadas quando apresentavam o primeiro trifólio expandido, porém, a inoculação foi realizada após 7 dias, quando elas haviam emitido o segundo trifólio.

As plantas inoculadas foram mantidas a $21^{\circ} \mathrm{C}$ e a $98 \%$ de umidade relativa por $48 \mathrm{~h}$. Na seqüência, elas foram transferidas para casa-devegetação onde permaneceram até a avaliação da severidade da doença realizada aos 7 dias após a inoculação (dai). Para avaliar o efeito local e residual utilizou-se a escala de notas de um a nove, descrita por Rava et al. (14), onde 1 significa ausência de sintomas e 9, maioria das plantas mortas pela doença. Para verificar o efeito sistêmico utilizouse a escala proposta por Tamayo (19) onde se avaliou apenas o segundo trifólio. Na escala de Tamayo (19) a nota 1 corresponde à ausência de sintomas e a nota máxima 9 , corresponde a necrose em folhas, pecíolos, ramos e gema apical cobrindo mais de $25 \%$ dos tecidos da planta.

Os 19 extratos foram subdivididos e testados em dois ensaios independentes, utilizando-se o delineamento inteiramente ao acaso, com cinco repetições, sendo considerado cada vaso com três plantas como uma repetição.

Após a verificação da homogeneidade das variâncias e teste de normalidade, procedeu-se a análise de variância e teste de separação de médias (Tukey, $\mathrm{Pd}<0,05$ ) para as variáveis significativas. $\mathrm{Na}$ primeira etapa selecionaram-se os extratos que reduziram significativamente a severidade da antracnose, em relação à testemunha.

Os extratos selecionados que promoveram efeito significativo local, residual e/ou sistêmico foram comparados em experimento delineado inteiramente ao acaso com cinco repetições. O cultivo das plantas, aplicação dos extratos e a inoculação foram realizados conforme descritos anteriormente. Porém, a severidade da antracnose foi avaliada além dos 7, também aos 12 dai.

Quanto ao efeito local, os extratos das macroalgas Acanthophora specifera, Bryothamnion seaforthii, Codium isthmocladum, Enteromorpha lingulata, e Sargassum stenophyllum reduziram significativamente a severidade de antracnose. A redução da severidade em plantas tratadas com estes extratos variou entre 17 e $30 \%$ em relação às plantas testemunhas (Tabela 1 ).

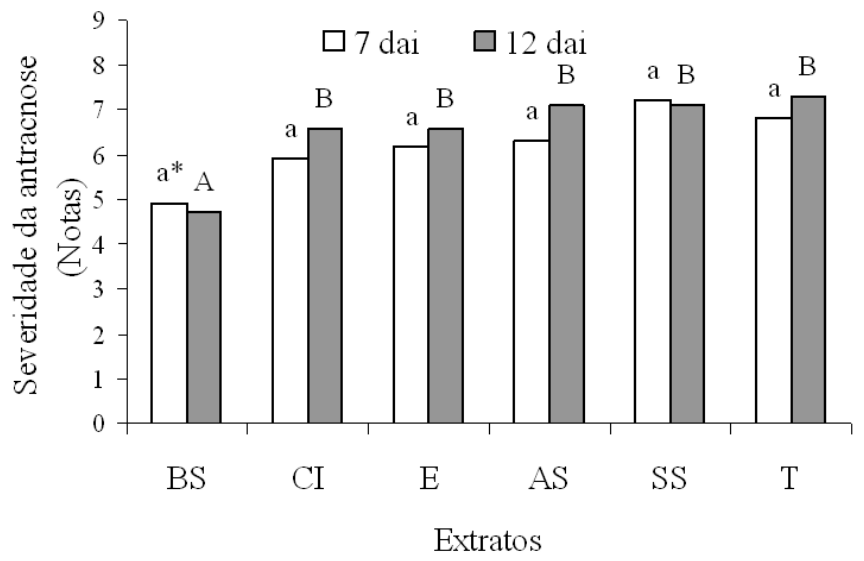

Figura 1. Efeito local de extratos etanólicos de macroalgas marinhas na severidade de antracnose do feijoeiro avaliada aos 7 e aos 12 dias após inoculação (dai). Legenda: Acanthophora specifera (AS); Bryothamnion seaforthii (BS); Codium isthmocladium (CI); Enteromorpha sp. (E); Sargassum stenophyllum (SS); Testemunha (T). *Barras seguidas pela mesma letra minúscula aos 7 dai e maiúscula aos 12 dai não diferem entre si pelo teste de Tukey $(\mathrm{P}>0,05)$.

Quanto ao efeito residual, os extratos das macroalgas Enteromorpha sp. e U. fasciata, bem como o da planta aquática Lemna sp., foram os que proporcionaram redução significativa na severidade de antracnose (Tabela 1). A redução da severidade de doença em plantas tratadas com estes extratos variou entre 9 a $24 \%$ em relação às testemunhas, que receberam uma nota média de severidade de 6,3 e 6,4 , nos ensaios 1 e 2 , respectivamente. A nota 6 , segundo a escala descritiva de Rava (14), indica a presença de manchas necróticas nas nervuras, perceptíveis em ambas as faces da folha e algumas lesões nos talos, ramos e pecíolos.

Quanto ao efeito sistêmico, somente o extrato de Lemna sp. reduziu significativamente a severidade da antracnose. Por outro lado, maiores severidades da antracnose no segundo trifólio foram observadas, quando as plantas foram pulverizadas com os extratos de $A$. specifera, C. isthmocladum, Gracilaria tepocensis e Spartina juncea.

Na comparação da eficiência de redução na severidade da antracnose pelos extratos selecionados observou-se redução significativa de $35 \%$ (Tukey, $\mathrm{P}<0,05$ ) da severidade da doença pela aplicação do extrato etanólico de B. seaforthii aos 12 dai (Figura 1). Já os extratos de $C$. isthmocladium, Enteromorpha sp., A. specifera e S. stenophyllum não afetaram significativamente a severidade da antracnose do feijoeiro em relação à testemunha.

Analisando-se o efeito residual dos extratos selecionados, verificouse redução de $22 \%$ da antracnose (Tukey, $\mathrm{P} \leq 0,05$ ) pela pulverização do extrato etanólico de U. fasciata aos 12 dai (Figura 2). Ao contrário dos resultados da seleção preliminar (Tabela 1), os extratos de Enteromorpha sp. e Lemna sp. não influenciaram a severidade da antracnose do feijoeiro em relação à testemunha.

Quanto ao efeito sistêmico, observou-se redução na severidade de doença no segundo trifólio aos 7 dai pelos extratos de Lemna sp. e $U$. fasciata na ordem de 55 e $44 \%$, respectivamente, em relação à testemunha. Esta redução da doença não foi, no entanto, mais significativa na segunda avaliação (Figura 2).

O extrato da alga vermelha $B$. seaforthii quando pulverizado em plantas de feijoeiro, quatro horas antes da inoculação reduziu em $35 \%$ a severidade da doença aos 12 dai em tecidos previamente tratados (Figura 1). Apesar de não afetar significativamente a severidade da 
antracnose aos 7 dai, o extrato promoveu redução no desenvolvimento da doença. Visto que a adição do extrato de $B$. seaforthii em meio de cultura, ocasiona uma forte redução no crescimento micelial de $C$. lindemuthianum (2), é provável que o extrato tenha afetado diretamente o fungo.

Alguns extratos com efeito local não apresentaram efeito residual, como por exemplo, os extratos de A. specifera, C. isthmocladum, E. lingulata, S. stenophyllum. Tal fato poderia ser explicado pela possibilidade de degradação dos compostos que atuaram na redução da doença no período entre o tratamento e a inoculação. Comportamento semelhante foi observado por Stadnik et al. (16) com a utilização de extratos de plantas no controle do oídio do pepino.

Os extratos de U. fasciata e Lemna sp., nos testes comparativos, reduziram sistemicamente as severidades de doença aos 7 dai em $44 \%$ e $55 \%$, respectivamente (Figura 2). Quando o extrato de $U$. fasciata foi pulverizado constatou-se ainda um efeito residual onde a severidade da antracnose foi reduzida em $24 \%$ aos 7 dai (Tabela 1) e em 24\% aos 12 dai (Figura 2). Considerando que o extrato da alga verde $U$. fasciata não apresentou efeito local, pode ter ocorrido a indução de resistência e para isto há, provavelmente, necessidade de um intervalo entre tratamento e inoculação. Este tipo de proteção tem sido atribuído a polissacarídeos algais capazes de elicitar reações de defesa da planta $(5,7)$.

A ação sistêmica do extrato da alga verde $U$. fasciata em plantas de feijoeiro contra a antracnose, encontrada neste trabalho, tem sido observada também em condições de campo por Loffaguen et al. (10). Ação de defesa sistêmica, proporcionada por polissacarídeos de Ulva spp, foi descrita contra C. trifolli em alfafa. Resposta esta, relacionada à formação de fitoalexinas, de proteínas relacionadas à patogênese (PRPs) e de proteínas formadoras de parede celular (5). Semelhantemente, a laminarina, obtida da alga marrom Laminaria digitata, também elicita respostas de defesa contra patógenos em plantas de fumo (7). Já Lizzi et al. (9) detectaram a síntese da fitoalexina capsidiol e atividade de peroxidase após a aplicação do extrato da alga marrom A. nodosum em plantas de videira e pimentão. Neste caso, ocorreu uma forte redução do míldio da videira (Plasmopara viticola) e da requeima do pimentão ( $P$. capsici) pela aplicação do extrato.

Alguns extratos, entre eles os de C. isthmocladum, G. tepocensis, Petalonia sp., S. juncea, estimularam a severidade de doença aos 7 dai, mesmo no trifólio não tratado. Tal fato pode indicar a inibição da ativação de mecanismos sistêmicos de defesa na planta, vindo a favorecer o estabelecimento da doença. Mudanças no metabolismo da planta devido à aplicação de extratos podem vir a suprimir componentes bioquímicos da resistência, como já observado por Stadnik \& Buchenauer (17).

As discrepâncias entre os resultados do testes de seleção e do teste comparativo dos extratos selecionados (Tabela 1 em relação às Figuras 1 e 2) podem ser explicadas pela influência de fatores ambientais. Mesmo as plantas sendo mantidas em casa-de-vegetação, onde se espera uma maior uniformidade, existem variações ambientais não passíveis de controle. Concomitantemente, Benevides et al. (3) afirmam que, além do componente genético, a composição química de macroalgas marinhas é também influenciada por fatores ambientais, como a salinidade, temperatura, intensidade de luz, concentração de nutrientes na água. Esses fatores ambientais podem afetar a resposta das plantas e a composição química das macroalgas. Tais diferenças poderiam explicar os diferentes resultados sob efeito residual e sistêmico apresentados entre algas de uma mesma espécie, ou de espécies distintas como, por exemplo, entre U. fasciata e U. lactuca.

O extrato de Lemna sp. promoveu fraca redução de severidade de doença sob efeito residual e um forte efeito sistêmico aos 7 dai (Tabela
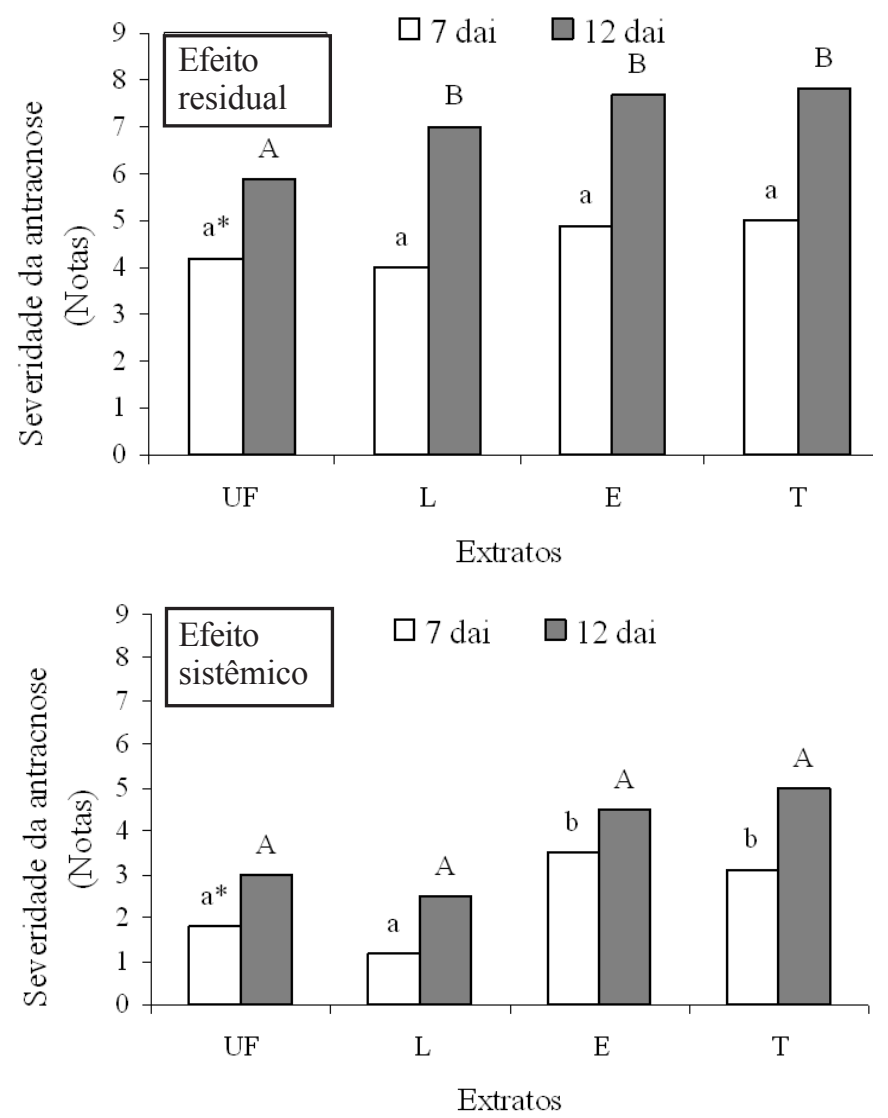

Figura 2. Efeito residual e sistêmico de extratos etanólicos de Ulva fasciata (UF), Lemna sp. (L) e Enteromorpha sp. (E) na severidade de antracnose avaliada aos 7 e aos 12 dias após inoculação (dai). Legenda: Testemunha (T); * Barras seguidas pela mesma letra minúscula aos 7 dai e maiúscula aos 12 dai não diferem entre si pelo teste de Tukey $(\mathrm{P}<0,05)$.

1 e Figura 2). Este é o primeiro relato que um extrato desta planta aquática é capaz de reduzir doença de planta.

Neste estudo bioprospectivo, conclui-se que as espécies promissoras foram $B$. seaforthii, Lemna sp. e U. fasciata. O extrato de $B$. seaforthii apresentou efeito local, provavelmente direto, reduzindo em $35 \%$ a severidade da antracnose, enquanto o extrato de $U$. fasciata demonstrou efeito residual com redução de $22 \%$. Somente os extratos de Lemna sp. e U. fasciata reduziram sistemicamente a severidade de doença aos 7 dai na ordem de 55 e $44 \%$, respectivamente, em relação à testemunha.

\section{AGRADECIMENTOS}

Os autores agradecem ao Prof. Dr. Paulo A. Horta Júnior do Departamento de Botânica da Universidade Federal de Santa Catarina pela identificação das macroalgas marinhas utilizadas neste trabalho.

\section{REFERÊNCIAS BIBLIOGRÁFICAS}

1. Abourriche, A.; Charrouf, M.; Berrada, M.; Bennamara, A.; Chaib, N.; Francisco, C. Antimicrobial activities and cytotoxicity of the 
brown alga Cystoseira tamariscifolia. Fitoterapia, Milão, v.70, n.6, p.611-614, 1999 .

2. Abreu, G.F. Bioprospecção de macroalgas marinhas e plantas aquáticas para o controle da antracnose (Colletotrichum lindemuthianum) do feijoeiro (Phaseolus vulgaris L.). 2005. 71f. Dissertação (Mestrado em Recursos Genéticos Vegetais) Universidade Federal de Santa Catarina, Florianópolis.

3. Benevides, N.M.B.; Sousa, E.; Silva, S.M.; Magalhaes, S.R.; Melo, F.R.; Freitas, A.L.P.; Vasconcelos, I.M. Proximate analysis, toxic and antinutritional factors of ten brazilian marine algae. Revista Brasileira de Fisiologia Vegetal, Campinas, v.10, n.1, p.3136, 1998.

4. Bennamara, A.; Abourriche, A.; Berrada, M.; Charrouf, M.; Chaib, N.; Boudouma, M.; Garneau, F.X. Methoxybifurcarerone: an antifungal and antibacterial meroditerpenoid from the brown alga Cystoseira tamariscifolia. Phytochemistry, Oxford, v.52, n.1, p.37-40, 1999

5. Cluzet, S.; Torregrosa, C.; Jacquet, C.; Lafitte, C.; Fournier, J.; Mercier, L.; Salamagne, S.; Briand, X.; Esquerré-Tugayé, M.T.; Dumas, B. Gene expression profiling and protection of Medicago truncatula against a fungal infection in response to an elicitor from green algae Ulva spp. Plant, Cell and Environment, London, v.27, p.917-928, 2004.

6. Goëmar. Laboratório do mar. Disponível em: <http::// www.goemar.com>. Acesso em: 20 dez. 2005.

7. Klarzynski, O.; Plesse, B.; Joubert, J.M.; Yvin, J.C.; Kopp, M.; Kloareg, B.; Fritig, B. Linear beta-1,3 glucans are elicitors of defense responses in tobacco. Plant Physiology, Rockville, v. 124, n.3, p.1027-1037, 2000

8. König, G.M.; Wright, A.D. Sesquiterpene content of the antibacterial dichloromethane extract of the marine red alga Laurencia obtusa. Planta Medica, Stuttgart, v.63, n.2, p.186-187, 1997.

9. Lizzi, Y.; Coulomb, C.; Coulomb, P.J.; Coulomb, P.O.; Polian, C. L'algue face au Mildiou: que avenir? Phytoma, Paris, v.508, p. 29-30, 1998.

10. Loffaguen, J.C.; Hartmann, O.E.L.; Talamini, V.; Stadnik, M.J. Extratos naturais no controle da antracnose e na produtividade do feijoeiro. Fitopatologia Brasileira, Brasília, v.29, supl., p.107, 2004. (Resumo).

11. Loffaguen, J.C.; Talamini, V.; Stadnik, M.J. Identificação da raça
73 de Colletotrichum lindemuthianum, agente causal da antracnose do feijoeiro, em dois municípios do Alto Vale do Itajaí, SC. Agropecuária Catarinense, Florianópolis, v.18, n.2, p. 87 $89,2005$.

12. López, B.C. Enzimas-Algas: posibilidades de su uso para estimular la produción agrícola y mejorar los suelos. Terra, Chapingo, v.17. n.3, p.271-276, 2000

13. Magallanes, C.; Córdova, C.; Orozco, R. Actividad antibacteriana de extractos etanólicos de macroalgas marinas de la costa central del Perú. Revista Peruana de Biología, Lima, v.2, n.10, p.125132, 2003.

14. Rava, C.A.; Molina, J.; Kauffman, M.; Briones, I. Determinación de Razas Fisiológicas de Colletotrichum lindemuthianum en Nicaragua. Fitopatologia Brasileira, Brasília, v.18, p.388-391, 1993.

15. Stadnik, M.J.; Zeferino, S.A.D.B. effect of marine macroalgae extracts on the severity of bean powdery mildew. Mitteilungen der Biologischen Bundesanstalt für Land- und Forstwirtschaft, Berlin, v.396, p.470, 2004.

16. Stadnik, M.J.; Bettiol, W.; Saito, M.L. Bioprospecting for plant and fungus extracts with systemic effect to control the cucumber powdery mildew. Journal of Plant Disease and Protection, Stuttgart, v.110, n.4, p.383-393, 2003.

17. Stadnik, M.J.; Buchenauer, H. Inhibition of phenylalanine ammonia-lyase suppresses the resistance induced by benzothiadiazole in wheat to Blumeria graminis f. sp. tritici. Physiological and Molecular Plant Pathology, New York, v.57, p. $25-34,2000$.

18. Talamini, V.; Stadnik, M.J. Extratos vegetais e de algas no controle de doenças de plantas. In: Stadnik, M.J.; Talamini, V. Manejo ecológico de doenças de plantas. Florianópolis: Universidade Federal de Santa Catarina, 2004. cap. 3, p.45-62.

19. Tamayo, P.J. Manejo y control de las enfermedades del fríjol voluble (Phaseolus vulgaris L.). Rionegro: Corporación Colombiana de Investigación Agropecuaria, 1995, 40p. (Boletín Técnico, n.4).

20. Vlachos, V.; Critchley, A.T.; Holy, A. Antimicrobial activity of extracts from selected southern African marine macroalgae. South African Journal of Science, Pretoria, v. 93, n. 7, p. 328-332, 1997 